\title{
Efficient estimation of eigenvalue counts in an interval
}

\author{
Edoardo Di Napoli ${ }^{*} \quad$ Eric Polizzi ${ }^{\dagger} \quad$ Yousef Saad ${ }^{\ddagger}$
}

August 6, 2014

\begin{abstract}
Estimating the number of eigenvalues located in a given interval of a large sparse Hermitian matrix is an important problem in certain applications and it is a prerequisite of eigensolvers based on a divide-and-conquer paradigm. Often an exact count is not necessary and methods based on stochastic estimates can be utilized to yield rough approximations. This paper examines a number of techniques tailored to this specific task. It reviews standard approaches and explores new ones based on polynomial and rational approximation filtering combined with a stochastic procedure.
\end{abstract}

\section{Introduction}

Recent efforts to develop alternative eigensolvers [27, 20, 26] for large scale scientific applications rely on "splitting" the spectrum of an eigenproblem in intervals and extracting eigenpairs from each one independently. In order to be efficient, this strategy requires an approximate knowledge of the number of eigenvalues included in each of these intervals. In general, any algorithm based on a form of subspace iteration for computing eigenvalues in a given interval benefits from the knowledge of the approximate number of eigenvalues inside that interval so as to select the dimension of the subspace to use in the iteration. In this context subspace size selection was already addressed by Sakurai and co-workers, [6, 28, 18], using a few different methods. An approximate count of the eigenvalues located in an interval can also help estimate the rank of a matrix and this is needed in the context of sampling-based methods [9, 19]. The goal of this paper is to explore inexpensive algorithms for determining the number of eigenvalues of a Hermitian matrix that are located in a given interval.

*Jülich Supercomputing Centre, Forschungszentrum Jülich, D-52425 Jülich. e.di.napoli@fz-juelich.de. Work of this author was supported by VolkswagenStiftung, the Excellence Initiative of the German federal and state governments and the Jülich Aachen Research Alliance High-Performance Computing.

${ }^{\dagger}$ Department of Electrical and Computer Engineering, University of Massachusetts, Amherst. polizzi@ecs.umass.edu. Work of this author was supported by the National Science Foundation under Grant \#ECCS-0846457.

${ }^{\ddagger}$ Computer Science \& Engineering, University of Minnesota, Twin Cities. saad@cs.umn. edu. Work of this author was supported by the Scientific Discovery through Advanced Computing (SciDAC) program funded by U.S. Department of Energy, Office of Science, Advanced Scientific Computing Research and Basic Energy Sciences DE-SC0008877 
The standard way of computing the number of eigenvalues of a Hermitian matrix $A$ located inside an interval $[a, b]$ is to resort to the Sylvester law of inertia [8]. For the sake of brevity this introduction will only discuss the standard eigenvalue problem although the paper deals with both standard and generalized problems. If $A$ is nonsingular, it admits the decomposition $A=L D L^{T}$, where $L$ is unit lower triangular, and $D$ is diagonal. The Sylvester inertia theorem then states that the inertias of $A$ and $D$ are the same. This means that the number of eigenvalues of $A$ that are positive is the same as the number of positive entries in the diagonal of $D$ (Sturm count). Thus, the $L D L^{T}$ factorizations for the shifted matrices $A-a I$ and $A-b I$ (assuming that these exist) yield respectively the number of eigenvalues larger than $a$ and $b$. The difference between these two numbers gives the eigenvalue count $\mu_{[a, b]}$ in $[a, b]$. While this method yields an exact count, it requires two complete $L D L^{T}$ factorizations and this can be quite expensive for realistic eigenproblems.

This paper discusses two alternative methods which provide only an estimate for $\mu_{[a, b]}$ but which are relatively inexpensive. Both methods work by estimating the trace of the spectral projector $P$ associated with the eigenvalues inside the interval $[a, b]$. This spectral projector is expanded in two different ways and its trace is computed by resorting to stochastic trace estimators, see, e.g.,[10, 31]. The first method utilizes filtering techniques based on Chebyshev polynomials. The resulting projector is expanded as a polynomial function of $A$. In the second method the projector is constructed by integrating the resolvent of the eigenproblem along a contour in the complex plane enclosing the interval $[a, b]$. In this case the projector is approximated by a rational function of $A$.

For each of the above methods we present various implementations depending on the nature of the eigenproblem (generalized vs standard), and cost considerations. Thus, in the polynomial expansion case, we propose a barrier-type filter when dealing with a standard eigenproblem, and two high/low pass filters in the case of generalized eigenproblems. In the rational expansion case we have the choice of using an LU factorization or a Krylov subspace method to solve linear systems. The optimal implementation of each method used for the eigenvalue count depends on the situation at hand and involves compromises between cost and accuracy. While it is not the aim of this paper to explore detailed analysis of these techniques, we will discuss various possibilities and provide illustrative examples.

The polynomial and rational expansion methods are motivated by two distinct approaches recently suggested in the context of electronic structure calculations: i) spectrum slicing and ii) Cauchy integral eigen-projection. In the spectrum slicing techniques [27] the eigenpairs are computed by dividing the spectrum in many small subintervals, called 'slices' or 'windows'. For each window a barrier function is approximated by Chebyshev-Jackson polynomials in order to select only the portion of the spectrum in the slice. In this method, it is important to determine an approximate count of the eigenvalue in each sub-interval in order to balance the calculations in a parallel implementation.

The second set of methods is based on eigen-projectors expressed in the form of Cauchy integrals [20, 26]. They essentially compute an orthonormal basis of the invariant eigenspace $\mathcal{V}$ associated with the eigenvalues located in the interval. For these methods to work efficiently one must have a good idea of the dimension of the subspace. This dimension must not be smaller than that of $\mathcal{V}$ if we are to account for all the eigenvalues inside the interval $[a, b]$ and, for reasons related to computational costs, it should also not be too large.

The paper is organized as follows. Section 2 introduces the eigenvalue count problem and 
gives an overview of traditional approaches for solving it. Section 3 discusses methods based on polynomial expansions and Section 4 is devoted to methods based on rational function expansions. Section 5 presents additional issues and provides a series of numerical tests to illustrate the behavior of the methods. Finally, Section 6 offers some concluding remarks.

\section{Eigenvalue counts}

Let $\lambda_{j}, j=1, \cdots, n$ be the eigenvalues, labeled by increasing value, and $u_{1}, u_{2}, \cdots, u_{n}$ the associated orthonormal eigenvectors of an Hermitian matrix $A$ (the generalized problem will be discussed later). Assuming that $\lambda_{1} \leq a<b \leq \lambda_{n}$, our aim is to count the number of eigenvalues $\lambda_{i}$ in the interval $[a, b]$. As described in the introduction, the standard way of obtaining this count is to resort to the Sylvester inertia theorem which will require two $L D L^{T}$ factorizations. Since exact factorizations can be computationally expensive, choosing the correct implementation of the $L D L^{T}$ factorization is crucial.

In modeling Hamiltonians of 2-dimensional (2-D) physical systems using finite differences sparse factorizations can be quite effective so that an eigenvalue count based on the inertias may be the method of choice. However, this approach becomes expensive in realistic cases where the matrix arises from simulations of 3-dimensional (3-D) phenomena. As is wellknown [7, 4], in the 3-D case the factorization becomes very costly both in terms of storage and arithmetic, due to the amount of fill-in generated. For dense eigenproblems the number of floating point operations per factorization is of order $O\left(n^{3}\right)$ and this becomes prohibitive for large matrices. Hence, counting eigenvalues based on the inertia theorem is a viable method only when dealing with fairly small dense matrices or for sparse matrices whose factorization is not too costly, e.g., those generated by 2-D models.

The problem of eigenvalue counts is also closely related to that of computing "Density of States" (DoS) a term used by physicists for the 'spectral density' or the probability of finding an eigenvalue at a given point in the real line. Some of these techniques bear some similarity with the ones described here [16]. For example, one can view the polynomial-based techniques presented in this paper as an adaptation of the Kernel Polynomial Method for computing the DoS to the problem of estimating eigenvalue counts [29, 33, 23, 30].

This paper explores two alternative approaches that compute an estimate of the eigenvalue count in the interval $[a, b]$ by seeking an approximation to the trace of the eigenprojector:

$$
P=\sum_{\lambda_{i} \in[a, b]} u_{i} u_{i}^{T} .
$$

The eigenvalues of a projector are either zero or one and so the trace of $P$ is equal to the number of terms in the sum (1), i.e. to the number of eigenvalues in $[a, b]$. Therefore, we can calculate the number of eigenvalues $\mu_{[a, b]}$ located in the interval $[a, b]$ by evaluating the trace of the related projector (1):

$$
\mu_{[a, b]}=\operatorname{tr}(P) .
$$

If $P$ were available explicitly, we would be able to compute its trace directly and obtain $\mu_{[a, b]}$ exactly. The projector $P$ is typically not available in practice but it is possible to inexpensively approximate it in the form of either a polynomial or a rational function of $A$. 
To this end, we can interpret $P$ as a step function of $A$, namely:

$$
P=h(A) \quad \text { where } \quad h(t)=\left\{\begin{array}{ll}
1 & \text { if } t \in[a, b] \\
0 & \text { otherwise }
\end{array} .\right.
$$

One can now approximate $h(t)$ with either a finite sum $\psi(t)$ of Chebyshev polynomials or a closed line integration of a rational function $\chi(t)$ on the complex plane. These two approaches lead to distinct approximations of the projector $P$, namely $P \approx \psi(A)$ or $P \approx \chi(A)$. In this form, it becomes possible to estimate the trace of $P$ by a so-called stochastic estimator developed by Hutchinson [10] and further improved more recently [31, 11, 34].

Hutchinson's unbiased estimator uses only matrix-vector products to approximate the trace of a generic matrix $A$. The idea is based on the use of identically independently distributed (i.i.d.) Rademacher random variables whereby each entry of randomly generated vectors $v$ assumes the values -1 and 1 with equal probability $\frac{1}{2}$. Hutchinson proved in a lemma that $E\left(v^{\top} A v\right)=\operatorname{tr}(A)$. Thus, an estimate $\mathcal{T}_{n_{v}}$ of the trace $\operatorname{tr}(A)$ can be obtained by generating $n_{v}$ samples of random vectors $v_{k}, k=1, . ., n_{v}$ and computing the average of $v_{k}^{\top} A v_{k}$ over these samples.

$$
\operatorname{tr}(A) \approx \mathcal{T}_{n_{v}}=\frac{1}{n_{v}} \sum_{k=1}^{n_{v}} v_{k}^{\top} A v_{k} .
$$

In practice there is no need to take vectors with entries equal to Rademacher random variables. Any sequence of random vectors $v_{k}$ whose entries are i.i.d. random variables will do as long as the mean of their entries is zero 3$]^{1}$. For example one can use normally distributed variables and define the Gaussian estimator exactly in the same fashion as in (3). While the variance of such an estimator is larger than that of Hutchinson, which uses Rademacher vectors, it shows a better convergence to the trace, in terms of the number of sample vectors $n_{v}[2]$.

Instead of the variance of the estimator, a more meaningful criterion is a less than $\delta$ probability that the estimator computes a value $\mathcal{T}_{n_{v}}$ for $\operatorname{tr}(A)$ whose relative error exceeds $\epsilon$, i.e.

$$
\operatorname{Pr}\left(\left|\mathcal{T}_{n_{v}}-\operatorname{tr}(A)\right| \geq \epsilon \operatorname{tr}(A)\right) \leq \delta .
$$

In the particular case the matrix $A$ is a projector $P$ its trace assumes only integers values and the rounded off value of $\mathcal{T}_{n_{v}}$ will be indistinguishable from $\operatorname{tr}(P)$ when their difference is less then $\frac{1}{2}$. In this particular case $\epsilon \equiv 1 /(2 \operatorname{tr}(P))$ and the following lemma holds [2]

Lemma 2.1 Let be $P \in \mathbb{R}^{n \times n}$ a projection matrix and let $\delta>0$ be a failure probability. Then for $n_{v} \geq 16 \operatorname{tr}(P) \ln \left(\frac{2}{\delta}\right)$, the estimator $\mathcal{T}_{n_{v}}$ of $P$ satisfies

$$
\operatorname{Pr}\left(\left\lfloor\mathcal{T}_{n_{v}}\right\rceil \neq \operatorname{tr}(P)\right) \leq \delta .
$$

The lemma above suggests that, even for $\delta=1$, the minimum number of sample vectors is of the order of magnitude of the trace of the projector with a large pre-factor. In practice the above lemma gives a rather loose bound and in many cases the estimator $\mathcal{T}_{n_{v}}$ converges for much lower values of $n_{v}$ than indicated by the lemma. In Sec. 5.4 we will show examples

\footnotetext{
${ }^{1}$ This form was used by physicists to compute the density of states [29, 33, 23, 30].
} 
for which there exists a value $n_{v} \lesssim \operatorname{tr}(P)$ after which the estimator $\mathcal{T}_{n_{v}}$ does not experience any relevant variation. In fact the source of error due to the use of a trace estimator is quite often negligible relative to the bias introduced by approximating the projector $P=h(A)$ with either $\psi(A)$ or $\chi(A)$.

We can now compute the trace of $P$ as:

$$
\mu_{[a, b]} \approx \begin{cases}\frac{n}{n_{v}} \sum_{k=1}^{n_{v}} v_{k}^{\top} \psi(A) v_{k} & \text { Polynomial expansion filtering } \\ \frac{n}{n_{v}} \sum_{k=1}^{n_{v}} v_{k}^{\top} \chi(A) v_{k} & \text { Rational expansion filtering. }\end{cases}
$$

where the sample vectors of the Gaussian estimator are normalized to one $\left\|v_{k}\right\|=1$. This constraint introduces a factor $n$ in the estimator but leaves the conclusion of lemma 2.1 unchanged. Moreover it avoids those rare cases when the Gaussian quotient returns rather large values. We will refer to this modified definition of $\mathcal{T}_{n_{v}}$ as Rayleigh Quotient (RQ) estimator.

The polynomial expansion approach does not require any factorization of $A$, and this is a big advantage when $A$ is large. Formally, the rational expansion approach would require a few such factorizations, one for each pole $z_{i}$ of the rational function. However, an exact factorization is no longer needed since we only need to solve with low accuracy linear systems with matrices of the form $A-z_{i} I$ and preconditioned iterative methods can be invoked for this purpose. In the next section we illustrate both approximations in detail and show that the error in computing $\mu_{[a, b]}$ depends essentially on how well the projector is approximated by the expansion and may be strongly affected by the presence of clustered eigenvalues close to the limits of the interval $[a, b]$.

\section{Polynomial expansion filtering}

In the polynomial filtering approach, the step function $h(t)$ in $(2)$ is expanded into a degree $p$ Chebyshev polynomial series:

$$
h(t) \approx \psi_{p}(t)=\sum_{j=0}^{p} \gamma_{j} T_{j}(t) .
$$

Here $T_{j}$ are the $j$-degree Chebyshev polynomials of the first kind, and the coefficients $\gamma_{j}$ are the expansion coefficients of the step function $h$ which are known to be

$$
\gamma_{j}=\left\{\begin{aligned}
\frac{1}{\pi}(\arccos (a)-\arccos (b)) & : \quad j=0, \\
\frac{2}{\pi}\left(\frac{\sin (j \arccos (a))-\sin (j \arccos (b))}{j}\right) & : \quad j>0 .
\end{aligned}\right.
$$

As a result we obtain an expansion of $P$ into matrices $T_{j}(A)$

$$
P \approx \psi_{p}(A)=\sum_{j=0}^{p} \gamma_{j} T_{j}(A) .
$$


The above derivation is based on the standard assumption that all the eigenvalues of $A$ lie in the interval $[-1,1]$ but it can be trivially extended to a generic spectrum with a simple linear transformation that maps $\left[\lambda_{1}, \lambda_{n}\right]$ into $[-1,1]$. This linear transformation is:

$$
l(t)=\frac{t-\left(\lambda_{n}+\lambda_{1}\right) / 2}{\left(\lambda_{n}-\lambda_{1}\right) / 2}
$$

and it requires estimates of the largest and smallest eigenvalues $\lambda_{n}, \lambda_{1}$. For the scheme to work it is necessary that the estimate for $\lambda_{n}$ be larger than $\lambda_{n}$ and the estimate for $\lambda_{1}$ be smaller than $\lambda_{1}$.

Two examples of a Chebyshev expansion of $h$ are shown (in red) in Fig. 1. As can be observed from the plots (red curves), the expansion of $h(t)$ has harmful oscillations near the boundaries. These are known as Gibbs oscillations. To alleviate this behavior it is customary to add damping multipliers - Jackson coefficients - so that (8) is actually replaced by

$$
P \approx \psi_{p}(A)=\sum_{j=0}^{p} g_{j}^{p} \gamma_{j} T_{j}(A)
$$

Notice that the matrix polynomial for the standard Chebyshev approach has the same expression as above with the Jackson coefficients $g_{j}^{p}$ all set to one, so we will use the same symbol to denote both expansions. The Jackson coefficients in their original form can be shown to be given by the formula,

$$
g_{j}^{p}=\frac{\left(1-\frac{j}{p+2}\right) \sin \left(\alpha_{p}\right) \cos \left(j \alpha_{p}\right)+\frac{1}{p+2} \cos \left(\alpha_{p}\right) \sin \left(j \alpha_{p}\right)}{\sin \left(\alpha_{p}\right)} \quad \text { where } \quad \alpha_{p}=\frac{\pi}{p+2},
$$

which was developed in [12]. Note that we can also write these coefficients in a slightly shorter form as:

$$
g_{j}^{p}=\frac{\sin (j+1) \alpha_{p}}{(p+2) \sin \alpha_{p}}+\left(1-\frac{j+1}{p+2}\right) \cos \left(j \alpha_{p}\right) .
$$

Substituting the expression for $\psi_{p}(A)$ directly into the stochastic estimator (5), yields the following estimate

$$
\mu_{[a, b]}=\operatorname{tr}(P) \approx \frac{n}{n_{v}} \sum_{k=1}^{n_{v}}\left[\sum_{j=0}^{p} \gamma_{j} v_{k}^{T} T_{j}(A) v_{k}\right]
$$

A clear advantage of this approach is that it requires only matrix-vector products. In addition, the vectors $w_{j}=T_{j}(A) v$ for a given $v$ can be easily computed using the 3 -term recurrence relation of Chebyshev polynomials $T_{j+1}(t)=2 t T_{j}(t)-T_{j-1}(t)$ which leads to

$$
w_{j+1}=2 A w_{j}-w_{j-1} .
$$

A similar method which uses a more complicated expansion into orthogonal polynomials, was also advocated in [24]. 


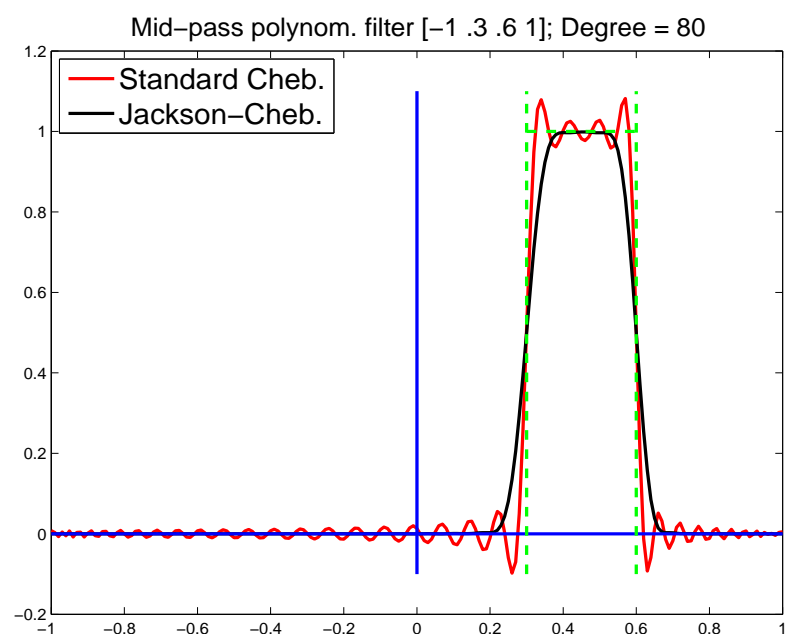

(a) Mid-pass filter of degree 80 .

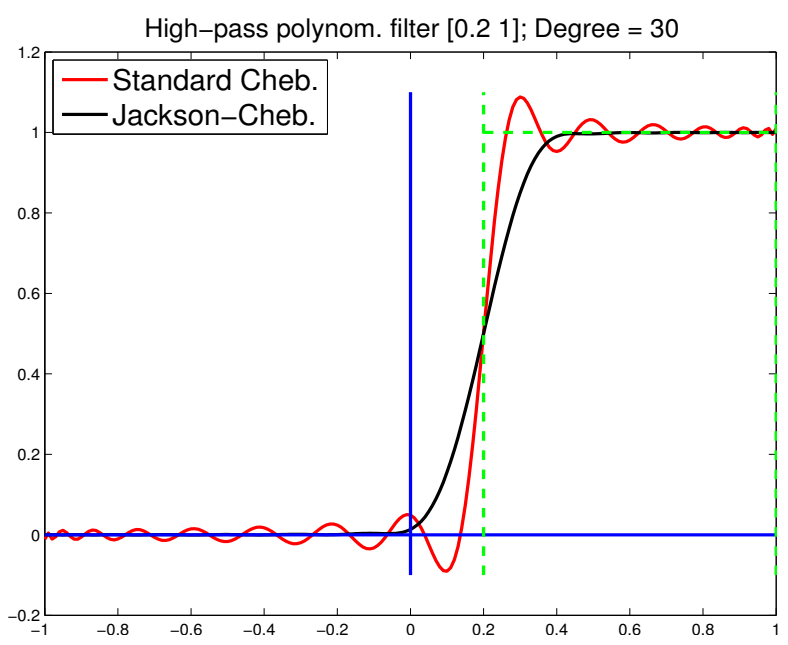

(b) High-pass filter of degree 30.

Figure 1: Two examples of polynomial filters.

\subsection{Theoretical considerations}

It is a known fact that the Chebyshev expansion of a function $h(t)$ (defined in $[-1,1]$ ) is essentially a Fourier expansion of a function obtained from $h$ by the change of variables $\cos \theta=t$ for $\theta \in[0, \pi]$. The function $h(t)$ becomes $h(\cos \theta)$ for $0 \leq \theta \leq \pi$ and this function is extended into the interval $(\pi, 2 \pi]$ by symmetry so the result is an even function (see [22, sec. 2.4] for details). When the function to expand is discontinuous, as is the case here, the Fourier expansion does not converge uniformly. In fact the function will oscillate around the discontinuity, and the maximum of the polynomial in $(-1,1)$ converges to a number strictly larger than one [1]. We do however, have pointwise convergence to $(h(x+0)+h(x-0)) / 2$ where $h(x+0)$ (resp. $h(x-0))$ represents the limit of $h(t)$ when $t$ converges to $x$ from the right (resp. left).

The Jackson expansion is a member of several techniques used to get rid of Gibbs oscillations. It can be viewed as the expansion of a sequence of smoothed versions of the original function. Another form of smoothing proposed by Lanczos [14, Chap. 4] and referred to as $\sigma$-smoothing, uses the simpler damping coefficients, called $\sigma$ factors by the author:

$$
\sigma_{0}^{p}=1 ; \quad \sigma_{j}^{p}=\frac{\sin \left(j \theta_{p}\right)}{j \theta_{p}}, \quad j=1, \cdots, p \quad \text { with } \theta_{p}=\frac{\pi}{p+1} .
$$

The damping factors are small for larger values of $j$ and this has the effect of reducing the oscillations. The Jackson coefficients have a much stronger damping effect on these last terms than the Lanczos $\sigma$ factors. For example the very last factors, and their approximate values for large $p$ 's, are in each case:

$$
g_{p}^{p}=\frac{2 \sin ^{2}\left(\alpha_{p}\right)}{p+2} \approx \frac{2 \pi^{2}}{(p+2)^{3}} ; \quad \sigma_{p}^{p}=\frac{\sin \left(p \theta_{p}\right)}{p \theta_{p}} \approx \frac{1}{p} .
$$

The above discussion underscores the difficulty in analyzing convergence and in selecting a proper polynomial degree. First we do not have uniform convergence for the case of the 
standard Chebyshev approximation and therefore it is difficult to relate the vectors $\psi_{p}(A) v$ to $h(A) v$ as would be required to obtain error bounds. Second, for the Jackson (and Lanczos $\sigma$-) smoothing the function being approximated is no longer just $h$ but a smoothed intermediate version $h^{(p)}$ which varies with the degree $p$ selected. So the process in this case has two steps: First smooth $h$ into $h^{(p)}$ then approximate $h^{(p)}$.

However, it is important to remember that the standard Chebyshev is the best approximation of the original function $h$. So any approximation to $h$ that is used, including the Jackson and Lanczos- $\sigma$ expansion, will have an error in the weighted $L_{2}$ norm that cannot be smaller that that of the standard Chebyshev approach. In the following we assume that the interval of interest is $[-1,1]$ and denote by $\|f(t)\|_{2}$ the $L_{2}$ norm associated with the Chebyshev inner product of functions:

$$
\left\langle\psi_{p}, \psi_{q}\right\rangle=\int_{-1}^{1} \frac{\psi_{p}(s) \psi_{q}(s)}{\sqrt{1-s^{2}}} d s
$$

We can now state the following result.

Proposition 3.1 Let $\psi_{p}^{(C)}, \psi_{p}^{(J)}$ and $\psi_{p}^{(L)}$ be the $p$-th degree Chebyshev, Jackson, or $\sigma$ Lanczos polynomial approximations to the function $h$ respectively, and let $\gamma_{j}$ be the sequence defined by (7) Then,

$$
\left\|\psi_{p}^{(C)}-h(t)\right\|_{2} \leq\left\|\psi_{p}^{(J)}-h(t)\right\|_{2}, \quad\left\|\psi_{p}^{(C)}-h(t)\right\|_{2} \leq\left\|\psi_{p}^{(L)}-h(t)\right\|_{2} .
$$

In addition,

$$
\left\|\psi_{p}^{(C)}-h(t)\right\|_{2}^{2}=\frac{\pi}{2} \sum_{j=p+1}^{\infty} \gamma_{j}^{2} \leq \frac{4 \pi}{3(p+1)}
$$

Proof. The two inequalities in (14) follow from the optimality of the least-squares approximation under the inner product (13). The first part of (15), i.e., the equality, follows from the fact that the scaled Chebyshev polynomials

$$
\sqrt{\frac{1+\delta_{j 0}}{\pi}} T_{j}(t)
$$

form a complete orthonormal sequence of polynomials ( $\delta_{i j}$ is the Kronecker delta) with $T_{j}$ having squared norm equal to $\pi / 2$ for $j>0$. For the second part, we have

$$
\begin{aligned}
\left\|\psi_{p}^{(C)}-h(t)\right\|_{2}^{2}=\frac{\pi}{2} \sum_{j=p+1}^{\infty} \gamma_{j}^{2} & =\frac{\pi}{2} \sum_{j=p+1}^{\infty}\left[\frac{2}{\pi}\left(\frac{\sin (j \arccos (a))-\sin (j \arccos (b))}{j}\right)\right]^{2} \\
& \leq \frac{\pi}{2} \sum_{j=p+1}^{\infty}\left[\frac{2}{\pi} \times \frac{2}{j}\right]^{2} \\
& =\frac{8}{\pi} \sum_{j=p+1}^{\infty} \frac{1}{j^{2}}
\end{aligned}
$$


Consider now the sum $\sum_{j=p+1}^{\infty} j^{-2}$. To simplify notation we first start the sum from $p$ instead of $p+1$. Then we have:

$$
\sum_{j=p}^{\infty} \frac{1}{j^{2}}=\sum_{l=1}^{\infty} \sum_{i=0}^{p-1} \frac{1}{(l p+i)^{2}} \leq \sum_{l=1}^{\infty} \sum_{i=0}^{p-1} \frac{1}{(l p)^{2}}=\sum_{l=1}^{\infty} p \frac{1}{(l p)^{2}}=\frac{1}{p} \sum_{l=1}^{\infty} \frac{1}{l^{2}}
$$

As is well-known $\sum_{l=1}^{\infty} l^{-2}=\pi^{2} / 6$. In the end we obtain:

$$
\sum_{j=p+1}^{\infty} \frac{1}{j^{2}} \leq \frac{1}{p+1} \sum_{l=1}^{\infty} \frac{1}{l^{2}}=\frac{1}{p+1} \frac{\pi^{2}}{6}
$$

Substituting this into (16) yields the inequality in 15 .

As indicated by the inequalities (14), we cannot do better than the standard Chebyshev polynomial if we are to measure the quality of the approximation by the $L_{2}$ norm based on the inner product (13). The convergence based on the bound 15 is like $1 / \sqrt{p}$, which is slow. However, much of the inaccuracy occurs around the jumps of the function. From a practical point of view, the polynomial is available explicitly and it is possible to analyze the error $\left|\psi_{p}(t)-h(t)\right|$ in specific subintervals to determine whether $\psi_{p}$ will be satisfactory. The cost of this analysis is trivial since the degree is usually moderate.

\subsection{Generalized eigenvalue problem}

We now consider the generalized eigenvalue problem $A x=\lambda B x$ where $A$ and $B$ are symmetric and $B$ is positive definite. In this case the projector $P$ in (1) becomes

$$
P=\sum_{\lambda_{i} \in[a b]} u_{i} u_{i}^{T} B
$$

and the eigenvalue count is again equal to its trace ${ }^{2}$. However, there are now two matrices involved and this projector does not admit an expression similar to that in (2) for the standard case. A common remedy to this issue is to compute the Cholesky factorization $B=L L^{T}$ of $B$ and transform the generalized eigenproblem into a standard one with the matrix $L^{-1} A L^{-T}$. This solution reintroduces the need for a costly factorization which we wanted to avoid in the first place. The following simple theorem yields the basis for an efficient alternative:

Proposition 3.2 Let $B$ be a semi-positive definite matrix and $B=L L^{T}$ its Cholesky factorization. Then the inertias of $A-\sigma B$ and $L^{-1} A L^{-T}-\sigma I$ are identical.

Proof. It is well-known that the inertias of a matrix $C$ and $X C X^{T}$ are the same for any nonsingular matrix $X$, see, e.g., [8]. The proposition follows by applying this result with $C=A-\sigma B$ and $X=L^{-1}$.

\footnotetext{
${ }^{2}$ Details on this can be found for example in 13 .
} 
A consequence of the above statement is that we can estimate the number of eigenvalues of the pair $(A, B)$ located in a given interval without resorting to any factorization. In essence the idea is to convert the eigenvalue count for the pair $(A, B)$ into two eigenvalue counts for two standard eigenvalue problems. Specifically, we have $\mu_{[a, b]}=\mu_{a}-\mu_{b}$ where $\mu_{a}$ is the number of positive eigenvalues of $A-a B$ and $\mu_{b}$ is the number of positive eigenvalues of $A-b B$.

Thus, this approach requires a Chebyshev expansion of the high-pass filters

$$
f_{\sigma}(t)= \begin{cases}1 & \text { if } t \geq \sigma \\ 0 & \text { otherwise }\end{cases}
$$

for $\sigma=a$ and $\sigma=b$ (see Fig. 1b, right, for an example). From these expansions, we would get estimates for the desired counts $\mu_{\sigma}$ for $\sigma=a, b$, i.e.,

$$
f_{\sigma}(t) \approx \sum_{j=0}^{p} \eta_{j}^{\sigma} T_{j}(t) \quad \rightarrow \quad \mu_{\sigma} \approx \sum_{j=0}^{p} \eta_{j}^{\sigma} \operatorname{tr}\left[T_{j}(A-\sigma B)\right] .
$$

Using the same set of sample vectors to estimate the two traces, we would then get the following eigenvalue count in the interval $[a, b]$ :

$$
\mu_{[a, b]}=\mu_{a}-\mu_{b} \approx \frac{n}{n_{v}} \sum_{k=1}^{n_{v}}\left[\sum_{j=0}^{p} \eta_{j}^{a} v_{k}^{T} T_{j}(A-a B) v_{k}-\sum_{j=0}^{p} \eta_{j}^{b} v_{k}^{T} T_{j}(A-b B) v_{k}\right] .
$$

As will be noted in the section devoted to the numerical experiments, for truly generalized problems $(B \neq I)$, the spectrum distribution of the matrices $A-\sigma B$ for $\sigma=a, b$, may lead to difficulties, requiring a very large degree polynomial in some cases. It is also possible to count eigenvalues to the left of $a$ and $b$ by using a low-pass filter. Notice that high-pass/lowpass filters require usually a lower degree than mid-pass ('barrier') filters, so this can also be used for the standard eigenvalue problem not just the generalized problem. As for the standard eigenvalue case, costly factorizations are avoided at the expense of using two filters with standard matrices.

\section{Rational expansion filtering}

A natural extension to the idea of polynomial filtering is to expand $P$ as a rational function. One of several ways of achieving this expansion is via the Cauchy integral definition of a projector:

$$
P=-\frac{1}{2 i \pi} \int_{\Gamma} R(z) d z
$$

where $R(z)=(A-z I)^{-1}$ is the resolvent of $A, z \in \mathbb{C}$, and $\Gamma$ is some smooth curve in the complex plane containing the desired part of the spectrum (see, e.g., [25]). Typically $\Gamma$ is taken to be a circle whose diameter is the line segment $[a, b]$. The above integral is then approximated by resorting to numerical integration methods, leading to

$$
P \approx \chi_{n_{c}}(A)=\sum_{j=1}^{n_{c}} \omega_{j}\left(A-z_{j} I\right)^{-1}
$$


where the $z_{j}$ 's are integration points and the $\omega_{j}$ s are quadrature weights. It is now possible to use the trace estimator by sampling with a set of random vectors. As shown in (5), the trace of $P$ will be approximated by the average of $(P v, v)$ over many sample vectors $v$, multiplied by $n$

$$
\mu_{[a, b]}=\operatorname{tr}(P) \approx \frac{n}{n_{v}} \sum_{k=1}^{n_{v}}\left[\sum_{j=1}^{n_{c}} \omega_{j} v_{k}^{T}\left(A-z_{j} I\right)^{-1} v_{k}\right] .
$$

This method is not new as a similar idea was advocated in [6].

\subsection{Approximations for the spectral projector}

The rational expansion can be easily adapted to the case of a generalized eigenvalue problem $A x=\lambda B x$. In this case the desired projector is still given by Eq. (18), but now the resolvent becomes

$$
R(z)=(A-z B)^{-1} B
$$

(see [13] for a simple derivation). This means that the only change from the standard case is that the resolvent $\left(A-z_{j} I\right)^{-1}$ in $(19)$ must be replaced by $\left(A-z_{j} B\right)^{-1} B$. In either case the stochastic estimation requires solving linear systems with multiple right hand sides for each integration point. In such situations it is customary to factorize the matrix $A-z_{j} B$ upfront so that the factors can be repeatedly used at a later stage. In general such factors do not need to be calculated exactly. For example, when employing an iterative procedure, an approximate factorization of the matrix $A-z_{j} B$ can be used as a preconditioner for the linear solver.

From the computational cost point of view, this approach may appear to be expensive and not competitive with the one based on Sylvester's inertia theorem described in Sec. 2 . Indeed, the inertia approach requires only two factorizations whereas we may now need a few such factorizations to get a good approximation to the spectral projector. In reality the method based on Sylvester's inertia must utilize an exact factorization, whereas in the above formula, all that is needed is to solve linear systems $\left(A-z_{j} I\right) y_{k}=v_{k}$ for many right-hand sides $v_{k}$ by any inexpensive procedure, including an iterative one.

The rational expansion approach should be favored when used in combination with the FEAST eigensolver [20, 5, 21] (or similar methods). Such an expansion allows us to get a rough eigenvalue count when the factorizations of $A-z_{i} B$ have been already computed in preparation for a subspace iteration-like procedure used by FEAST for the symmetric problem. In such procedure Gauss-quadrature points (i.e. shifts $z_{i}$ ) are positioned along the half-circle contour as shown on the left of Fig. 2. The same quadrature points can be used to compute the rational approximation for the projector $\chi_{n_{c}}$ which is plotted, as a function of $\lambda$, on the right side of Fig. 2. Note the rapid decay of the rational function from $\simeq 1$ in the middle of the interval $[-1,1]$ to $\simeq 0$ outside, which both explain the expected efficiency of the trace estimator (19), and can lead to some remarkable convergence rates for the FEAST subspace iteration procedure. More precisely, for the eigenpair $\left(\lambda_{j}, u_{j}\right)$, the error introduced by the numerical integration on the projector is bounded by (Theorem 4.1 in [32]):

$$
\left\|\left(h-\chi_{n_{c}}\right) u_{j}\right\|_{B} \leq \alpha\left|\frac{\chi_{n_{c}}\left(\lambda_{M_{0}+1}\right)}{\chi_{n_{c}}\left(\lambda_{j}\right)}\right| \quad \forall j \in 1, \ldots, \mu_{[a, b]}
$$




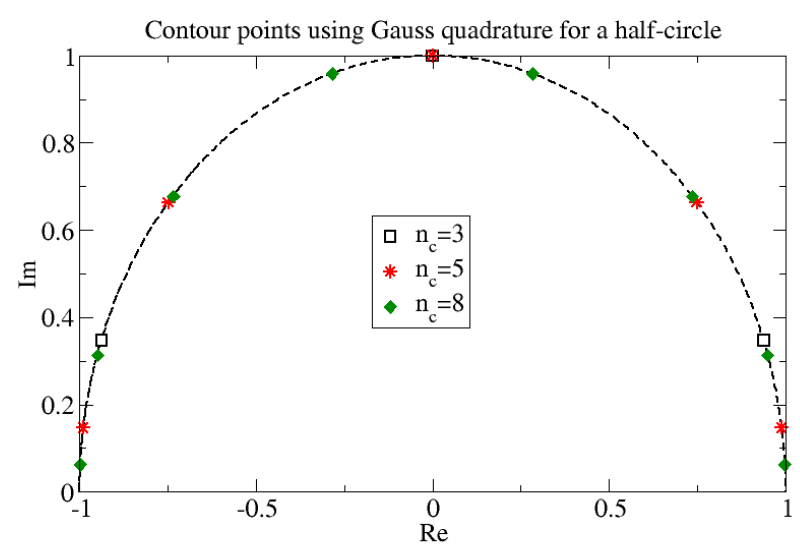

(a) Quadrature points.

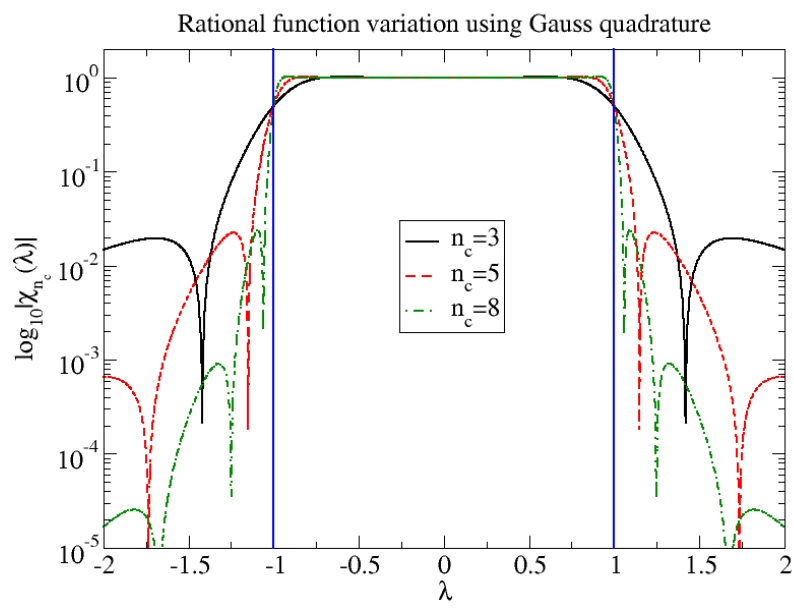

(b) Rational approximation filter.

Figure 2: Position in the complex plane of the $n_{c}$ Gauss quadrature points using an integration along the half-circle, and the corresponding values of the rational function $\chi_{n_{c}}(\lambda)$ using a semi-log plot. The search interval is here set to $[-1,1]$.

where the first $\mu_{[a, b]}$ eigenvalues $\lambda$ are the ones inside the search interval $[-1,1]$, and $M_{0}$ is the size of the search space. The ratio between the rational functions in 20 is inversely proportional to the FEAST convergence rate, and it is expected to decrease exponentially with the number of integration points $n_{c}$. In turn, the value of this ratio may increase significantly in case one or both of the search interval boundaries is (are) near clusters of the spectrum (i.e. if $M_{0}$ is too close to $\mu_{[a, b]}$ for FEAST). Similarly, the eigenvalue count may not be very accurate in this case (see Sec. 5.3 for more details).

\subsection{Practical considerations}

Next we consider a few implementation issues related to the rational approximation filtering approach. The eigenvalue count estimator is based on the formula

$$
\operatorname{tr}(P) \approx \frac{n}{n_{v}} \sum_{k=1}^{n_{v}} \sum_{j=1}^{n_{c}} \omega_{j} v_{k}^{T}\left(A-\sigma_{j} I\right)^{-1} v_{k} .
$$

The above formula involves two loops: the $k$-loop which we will refer to as the 'sample vector loop' and a $j$ loop which we call the 'integration loop' with $n_{c}$ the number of integration points. As it is written, the above formula suggests that we would run a vector loop, in which we would generate random vectors, then for each vector in turn we would solve $j$ right-hand sides (integration loop). This is fine when a direct solver is used for the solutions, provided we store the factorizations for each integration point.

An important observation here is that we can also swap the two loops, in effect exploiting 
the fact that the trace of the sum of operators is the sum of the different traces:

$$
\begin{aligned}
\operatorname{tr}(P) & \approx \operatorname{tr} \sum_{j=1}^{n_{c}} \omega_{j}\left(A-\sigma_{j} I\right)^{-1} \\
& =\sum_{j=1}^{n_{c}} \omega_{j} \operatorname{tr}\left(A-\sigma_{j} I\right)^{-1} \\
& \approx \frac{n}{n_{v}} \sum_{j=1}^{n_{c}} \omega_{j} \sum_{k=1}^{n_{v}} v_{k}^{T}\left(A-\sigma_{j} I\right)^{-1} v_{k} .
\end{aligned}
$$

This can be quite useful in a processing phase: As each of the $n_{c}$ factorizations is obtained we generate a number of random vectors and estimate the trace of $\left(A-\sigma_{j} I\right)^{-1}$ with them. At the end of all the factorizations, we end up with an eigenvalue count estimate which can be exploited to determine the subspace dimension to use in the FEAST subspace iteration procedure (i.e. value of $M_{0}$ in $(20)$ ).

Iterative solvers offer an appealing alternative to exact factorizations. When using a Krylov subspace method without preconditioning, one can immediately make the well-known observation that the various systems $\left(A-\sigma_{j} I\right) y_{j}=v$ for different $j$ 's and for each random vector $v$, can all be solved with the same Krylov subspace (e.g. [17]).

Another issue is to determine what accuracy to require from the solver. In the context of the FEAST eigensolver [5], the residual norm criterion for GMRES will clearly yield a similar size error for the eigenvector as it was observed and analyzed in [13, 32]. The problem for counting eigenvalues is slightly different. A first observation is that we are not interested in computing eigenvalues or eigenvectors. It is well-known that the error made on the eigenvalues is typically of the order of the square of the related residual norm [25]. From our observations, a high accuracy is not needed. However, there is a minimum accuracy required, below which the method will no longer work. It is also important to have consistent error thresholds. For example, just using a fixed number of GMRES steps will usually not work. It is best to use a criterion based on a residual norm reduction, for example by a factor of $10^{-2}$.

\section{$5 \quad$ Numerical experiments}

This section provides numerical illustrations of a number of features and discusses additional issues of the methods proposed in Sec. 3 and 4.

\subsection{Polynomial filtering: Standard and Jackson polynomials}

We use as first example the Na5 matrix generated by the PARSEC code. This matrix, available from the University of Florida matrix collection 3 , is of size $n=5832$ and has $n n z=305630$ nonzero entries. We computed eigenvalues at the outset and defined the interval $[a, b]$ so that $a$ is in the middle of $\lambda_{100}, \lambda_{101}$, and $b$ is in the middle of $\lambda_{200}, \lambda_{201}$. In this situation the exact eigenvalue count is 100 . Using $n_{v}=30$ and a degree 70 standard

\footnotetext{
$\sqrt[3]{\text { http://www.cise.ufl.edu/research/sparse/matrices/ }}$
} 

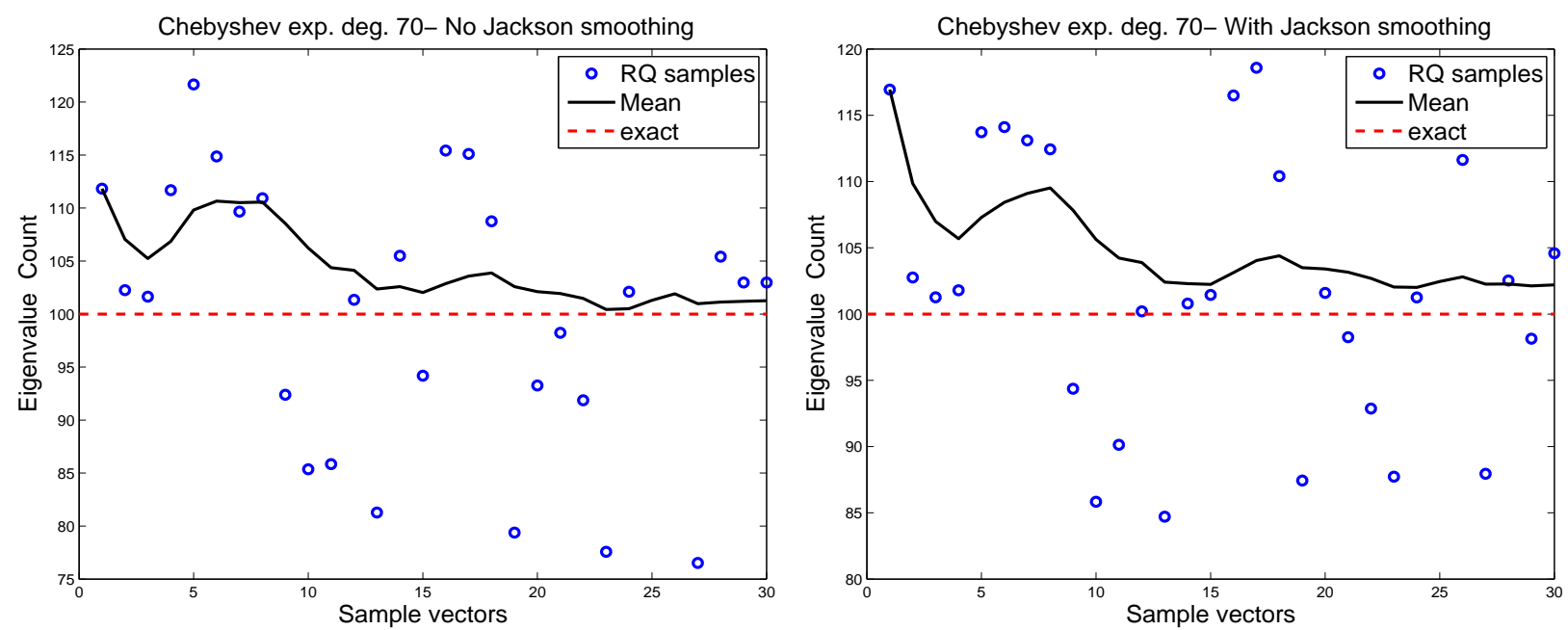

Figure 3: Chebyshev vs Jackson-Chebyshev for counting eigenvalues $\lambda_{101}$ to $\lambda_{200}$ for the matrix Na5.

Chebyshev polynomial yields the results shown on the left plot of Fig. 3. The right plot of the same figure shows the result obtained with Jackson-Chebyshev polynomials of the same degree: the last value of the computed average (eigenvalue count estimate) was 101.25 for Chebyshev and 102.20 for Jackson-Chebyshev. The same sequence of random vectors were used in both Chebyshev and Jackson-Chebyshev.

From this specific example, one may conclude that Jackson tends to often give an overestimate whereas standard Chebyshev often gives an underestimate. In reality, the behavior of both methods depends crucially on the eigenvalue density distribution in relation to the position of the extrema of the interval $[a, b]$ and the situation is often reversed (see next subsection).

\subsection{Estimate bias}

In this section we consider only polynomial methods, although similar statements can be made for the rational approximation methods. Since $\psi_{p}(A)$ is only an approximation to the projector (1), its trace will not be equal to the number of eigenvalues inside the interval. In some situations (not involving clustering) a relatively large degree is needed to get a reasonable approximation. An illustration of what can happen when the degree is not large enough is shown in Fig. 4. On the left side the lower horizontal line is the trace of the matrix $\psi_{p}(A)$ computed with a low degree. The higher dashed horizontal line is the actual eigenvalue count. There is a substantial gap between the two showing that the trace estimator does work, but it estimates a trace of an inaccurate projector. The right side of the figure shows that the gap narrows substantially for a higher degree polynomials. As shown in Table 1, a degree above 70 is necessary to get an approximation that is close enough, where the lower and upper dashed lines are close. In this regard there could be a big difference between the Jackson-Chebyshev and the standard Chebyshev polynomials. Here Jackson smoothing seems to be very detrimental to the estimation. In other situations, the Jackson polynomial 
performs better.
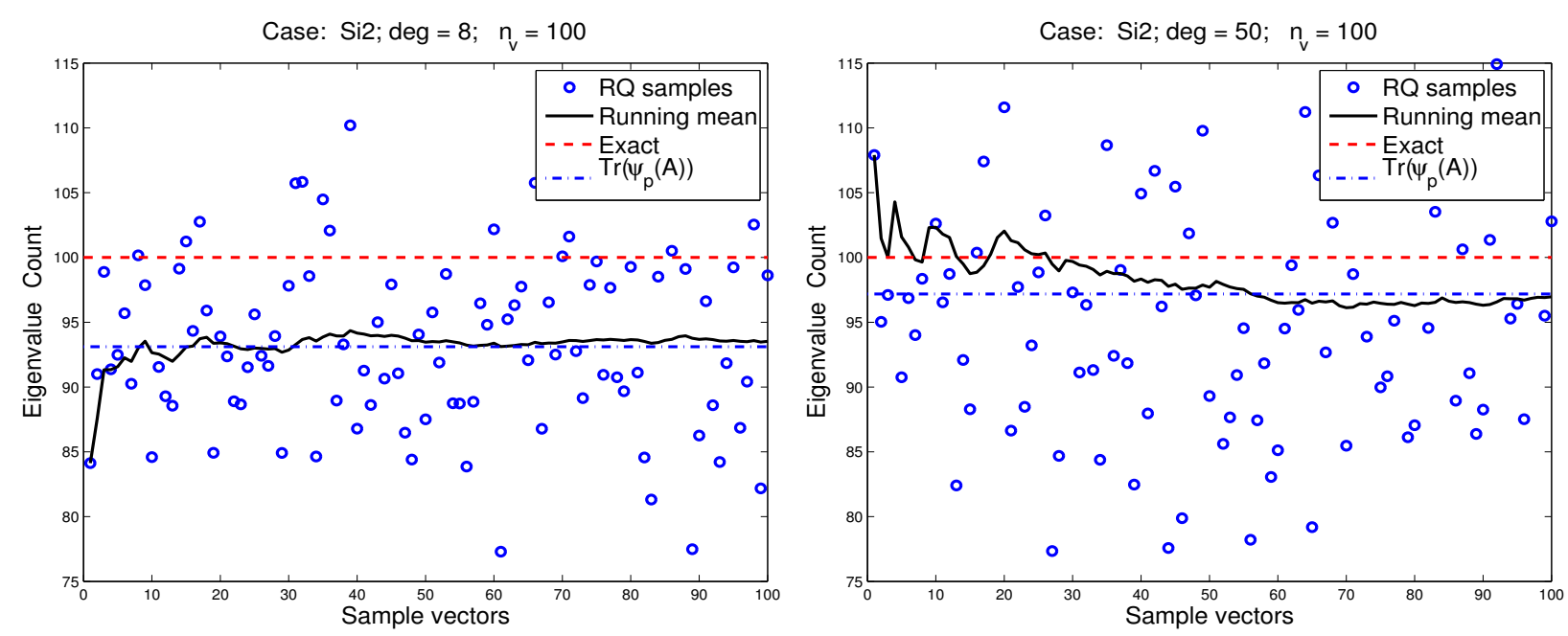

Figure 4: Chebyshev based counting with a degree 8 (left) and 50 (right) for matrix Si2.

We explored a few ways to fix this bias. In particular the simplest correction is to compare the integrals of $\psi_{p}(t)$ in $[-1,1]$ with that of the step function on the same interval. The integral of the step function is just $b-a$. The integral of each $T_{k}$ in the interval $[-1,1]$ is readily computable (it is equal to zero when $k$ is odd and to $-2 /\left(k^{2}-1\right)$ when $k$ is even). Then one can obtain the integral of $\psi_{p}$ in $[a, b]$ from which a corrective factor can be obtained. However, experiments with such a correction were mixed. The difficulty inherent to this problem is that the bias will certainly depend on the distribution of eigenvalues.

\begin{tabular}{|l|r|r|r|r|r|r|r|r|}
\hline$p$ & 8 & 20 & 30 & 40 & 50 & 70 & 100 & 120 \\
\hline $\operatorname{tr}\left[\psi_{p}^{(C)}(A)\right]$ & 93.12 & 97.29 & 96.98 & 96.81 & 97.19 & 101.58 & 101.54 & 100.76 \\
\hline $\operatorname{tr}\left[\psi_{p}^{(J)}(A)\right]$ & 74.53 & 89.59 & 93.00 & 94.51 & 95.29 & 95.97 & 96.99 & 97.74 \\
\hline
\end{tabular}

Table 1: Evolution of the trace of $\psi_{p}(A)$ for the standard Chebyshev $\psi_{p}^{(C)}$ and JacksonChebyshev $\psi_{p}^{(J)}$ approaches for the Si2 test case. The exact count is 100 .

\subsection{Eigenvalue clustering}

As was mentioned at the end of Sec. 5.1, the accuracy of the eigenvalue estimate depends on the eigenvalue distribution of $A$. In particular, if either $a$ or $b$ is close or inside a cluster of eigenvalues, $\mu_{[a, b]}$ may overestimate or underestimate the true number of eigenvalues in $[a, b]$. Moreover the extent of the error is related to both the size of the cluster and the relative distance $D_{\lambda}=\frac{\left\|\lambda_{i+1}-\lambda_{i}\right\|}{\left\|\lambda_{i}\right\|}$ between eigenvalues in the cluster.

In order to verify this point we ran a series of tests on an eigenproblem containing artificially engineered clusters of distinct length and density. For each cluster we placed 
the rightmost ending of the interval $[a, b]$ either at the beginning or at the end of the set of values forming the cluster. For each case we computed $\mu_{[a, b]}$ using both the simple

Chebyshev $\psi_{p}^{(C)}(A)$ and the Jackson-Chebyshev expansion $\psi_{p}^{(J)}(A)$ with two quite different polynomial degrees $p$.

Results from our tests clearly show that when the filtering interval intersects the beginning of a cluster both $\psi_{p}^{(C)}(A)$ and $\psi_{p}^{(J)}(A)$ overestimate the eigenvalue count. In contrast when the interval intersects the end of a cluster both expansions underestimate the eigenvalue count. This result does not in general depend on the polynomial degree adopted and reflects the tailing effects of the expansions as shown for example in Fig. 1a. Moreover we observed that the extent of the error made depends on the length of the cluster and on the relative distance between eigenvalues in the cluster.

We repeated the set of tests for the rational expansion and observed a behavior similar to polynomial expansions. When most of the values characterizing a cluster are close but outside the interval $[a, b]$, the trace of $\chi_{n_{c}}(A)$ overestimates the number of eigenvalues. In contrast, if such cluster of values is almost entirely within the filtering interval, the eigenvalue count is underestimated. In other words, clusters located near the end-points will just exacerbate the bias described in section 5.2 .

\subsection{Number of sample vectors and estimator accuracy}

According to lemma 2.1 there exist a minimum value of vectors for which we can assert we converged to the value of the trace with probability $1-\delta$. While this bound is rather loose it indicates that convergence is reached for $n_{v} \sim \mathcal{O}(\operatorname{tr}(P))$ for any meaningful $\delta$. In this section we illustrate the numerical behavior of the estimator with two typical examples which we briefly comment.

In both plots of Fig. 5 the jagged lines representing the estimates quickly reduce their wide oscillations and remain close to the exact trace of the approximated $P$. The value of $n_{v}$ for which the range of the oscillations from one sample vector to the next become consistently less than one is between $50 \leq n_{v} \leq 100$ for Fig. $5 \mathrm{a}$ and $200 \leq n_{v} \leq 300$ for Fig. 5b. These two typical examples illustrate that, for intervals containing a small number of eigenvalues, a minimum number of vectors $n_{v} \sim \operatorname{tr}(P)$ may be needed. In fact a good estimate for the trace is based on having enough sample vectors $v_{k}$ to represent almost entirely the subspace generated by the eigenpairs in the interval $[a, b]$ and such a number is exactly $\operatorname{tr}(P)$.

When the number of eigenvalues in $[a, b]$ is larger than a few dozens, the necessary number of sample vectors can often be lower $n_{v} \lesssim \operatorname{tr}(P)$. In general, for the same approximated projector, the minimum value of $n_{v}$ varies slightly every time a different set of sample vectors is selected, but overall the experimental bound differs from the theoretical one given by lemma 2.1 having a much smaller value for the pre-factor of $\operatorname{tr}(P)$.

While this conclusion seems natural due to the nature of the expansions, it may introduce a practical difficulty in estimating a priori the minimum number of sample vectors necessary for the estimation of the trace. This difficulty can be overcome by computing the estimator incrementally and monitoring the value of the increment over a small range of previous samples (typically no more than 10). When such an increment remains substantially constant and less than one, the estimated value for the trace can be safely said to have converged. 
Eig: Si2; $\operatorname{deg}=50 ; n_{c}=8 ; n_{v}=300$

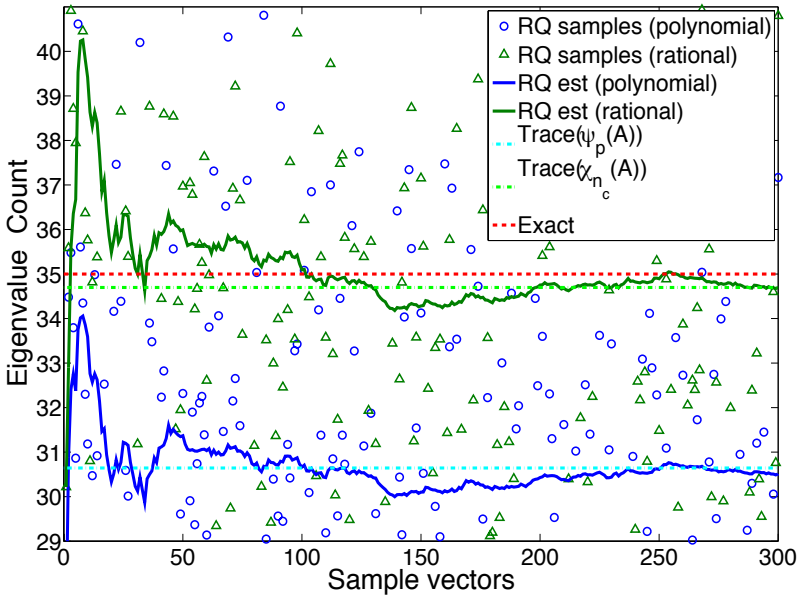

(a) Small interval $[a, b]$ including $\lambda_{5}$ up to $\lambda_{40}$.
Eig: $n$ n5; $\quad$ deg $=50 ; \quad n_{c}=8 ; \quad n_{v}=700$

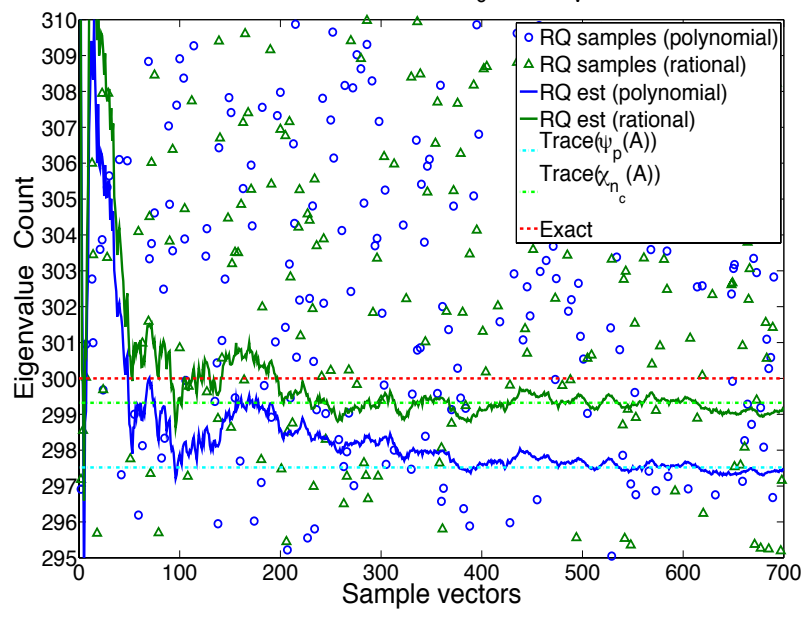

(b) Large interval $[a, b]$ including $\lambda_{100}$ up to $\lambda_{400}$.

Figure 5: Behavior the Rayleigh Quotient estimator when the number of sample vectors $n_{v}$ varies. Two distinct test cases are considered with both the polynomial and rational expansion techniques. (a): Case of a small problem $(n=789)$ and an interval containing a small number of eigenvalues. (b): Both the system size and number of eigenvectors are one order of magnitude larger. In each plot the random generated vectors are the same for both estimators and the straight color lines are the exact traces of the approximate projector.

\subsection{Rational approximation filtering: Direct vs iterative solvers}

We ran tests with the same Na5 example which was used in Sec. 5.1. When employing exact factorizations for each integration point and $n_{v}=40$ sample vectors, the eigenvalue count is $\mu_{[a, b]}=98.64$ for $n_{c}=3$ and $\mu_{[a, b]}=100.27$ for $n_{c}=5$. Clearly more accurate quadrature rules will yield more accurate counts. Note however, that much faster results can be obtained using the Sylvester inertia approach since this requires only two direct factorizations (in real arithmetic).

We can avoid costly direct factorizations and make use of iterative solvers to improve efficiency. For the Na5 example with same parameters as above, we solved the linear systems using GMRES with residual norm equal to $10^{-1}, 10^{-2}$ and $10^{-3}$ and obtained a trace estimate equal respectively to 75.35, 97.61 and 98.65. As mentioned in Sec. 4.2 a minimum accuracy for the iterative solver is necessary but we observe that the estimates are reasonably good starting with a high value for the residual threshold (e.g. $10^{-2}$ for GMRES) when compared with the direct case. Using $n_{c}=3$, Fig. 6 shows the absolute errors on $\mu_{[a, b]}$ obtained by comparing direct factorizations and GMRES with $10^{-2}$ and $10^{-3}$ residual threshold. Results based on the iterative solver are found in extremely good agreement with those of the direct factorization, and this agreement is expected to be preserved independently of the number of integration points.

When there is no preconditioning, the iterative procedure requires only matrix-vector multiplications with the matrix $A$. Therefore, using an iterative solver is a promising method for handling very large systems. In order to further enhance the performance, one can think of two options to be developed: (i) the generation of a single Krylov subspace common to 
all integration points; (ii) the use of (cheap) preconditioners.

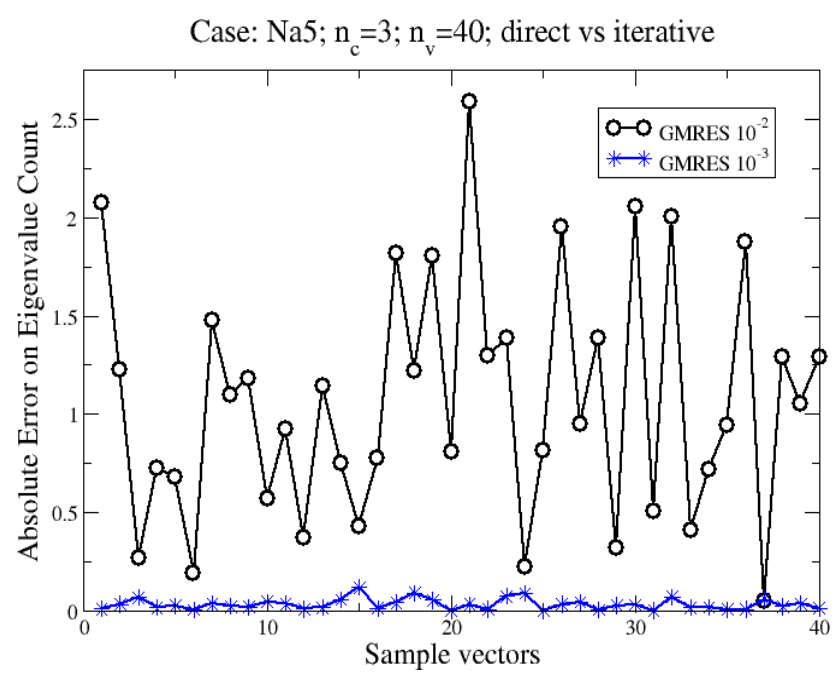

Figure 6: Absolute errors on the eigenvalue count between results obtained with direct factorization and GMRES using both $10^{-2}$ and $10^{-3}$ convergence criteria. This absolute error (i.e. $\left|\mu_{[a, b]}^{\text {Direct }}-\mu_{[a, b]}^{G M R E S}\right|$ ) decreases to zero using smaller GMRES residual thresholds.

We also tested the polynomial and rational filtering methods on a generalized eigenvalue problem corresponding to a 2-D FEM simulation matrix and available from the FEAST package [5]. In this case the matrices $A, B$ have size $n=12,450$. The number of nonzero entries in both $A$ and $B$ is $n n z=86,808$. The number of eigenvalues inside the desired interval is 100 (i.e. 100 lowest eigenvalues). On the left plot of Fig. 7 the varying average of $\mu_{[a, b]}$, obtained with a degree 100 polynomial filtering, shows an almost perfect agreement with the exact count.

For the rational filtering method we run tests with $n_{c}=5$, using a direct solver and GMRES with and without preconditioning (denoted by GMRES and P-GMRES respectively). Comparison of these approaches are shown on the right plot of Fig. 7. For clarity the figure now omits the small circles corresponding to Rayleigh quotients of each sample and shows only the running mean. We tested the preconditioned GMRES with an ILU factorization using a drop tolerance of droptol $=0.01$ and a pivoting threshold of 0.05 as defined by the MATLAB ilu function with its default reordering. For all the runs with P-GMRES we used a restart dimension of 20 and limited the number of steps to 200. The iteration is stopped when the residual norm drops by a preset threshold tolerance (tol). Results with tol $=10^{-2}$ are shown. Other results obtained with GMRES and P-GMRES with tol $=10^{-3}$ were quasi-identical with those obtained with the direct solver and are omitted.

The curves show an underestimation of the eigenvalue counts for both GMRES and PGMRES. Remarkably, each of these two curves deviates from that of the exact solver by nearly a constant, indicating the existence of a bias for the case of insufficient accuracy. This observation is not easy to explain and merits further investigation.

The better accuracy afforded by the rational approximation approach comes at a high memory cost. The total number of nonzero elements for all the LU factorizations generated for the 5 integration points for this case is $n n z_{\text {LUtotal }}=22,239,530$, an enormous amount relative to the original number of nonzero entries which is under 100,000 . In contrast, when 
the ILU factorization is used for $n_{c}=5$ and under the conditions of the above experiment the total number of nonzero elements used for all $5 \mathrm{ILU}$ factorizations drops to $n n z_{L U t o t a l}=$ 799,143 , about 28 times smaller. This is a 2-D problem. For a 3-D problem a direct approach becomes unfeasible on a standard workstation and using a preconditioned iterative solver is the only option.
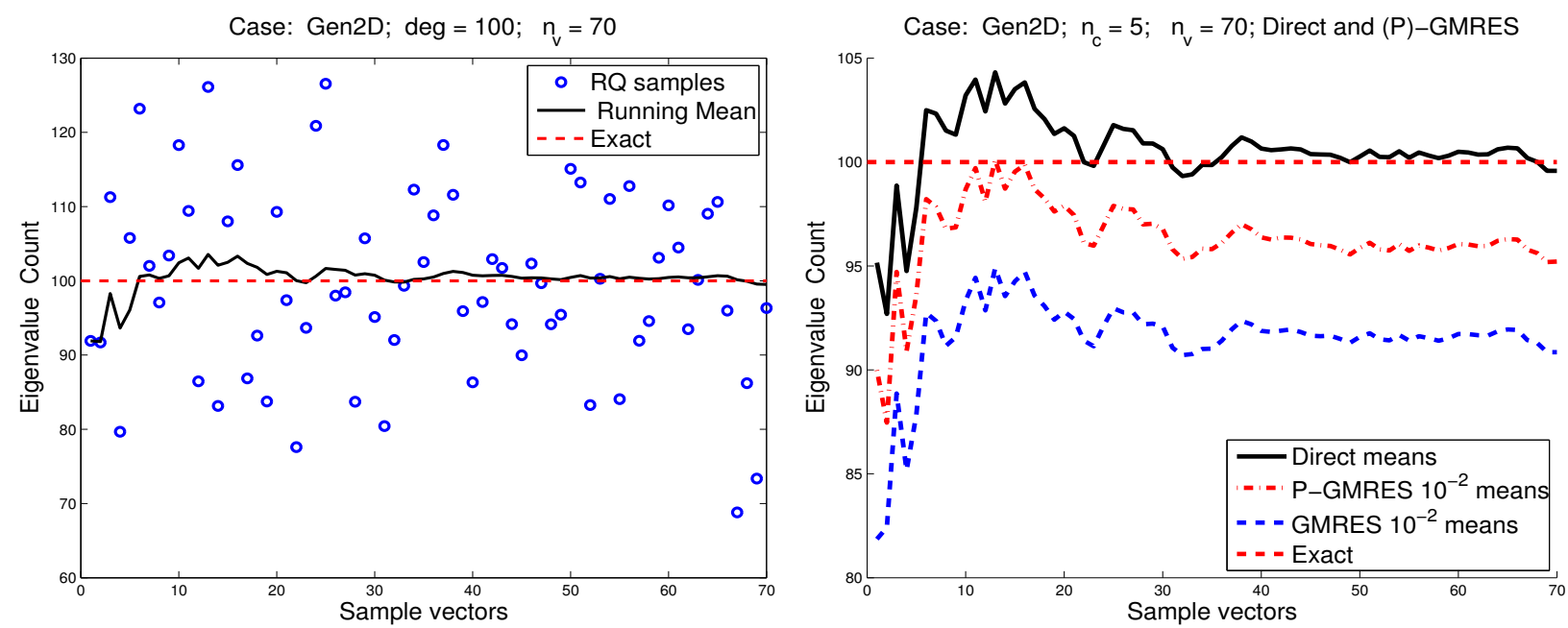

Figure 7: (Standard) Chebyshev vs rational approximation for the Gen2D matrix. The sequences of random vectors used in all the experiments are identical.

\subsection{Using estimated eigenvalue counts in FEAST}

As mentioned in Sec. 4.1, the convergence rate of the FEAST subspace iterations mainly depends on the size $M_{0}$ of the search subspace and the value of the rational function at $\lambda_{M_{0}+1}$ (i.e. $\chi_{n_{c}}\left(\lambda_{M_{0}+1}\right)$ in (20)). In Fig. 2, for example, we note for the case $n_{c}=8$ and the search interval $\lambda \in[-1,1]$ that the rational function is equal to $\sim 10^{-4}$ for $\lambda= \pm 1.5$. As a result, if the search subspace size $M_{0}$ is taken large enough to include all eigenvalues between $[-1.5,1.5]$, one can expect the residuals of the eigenpairs within $[-1,1]$ to converge with the same (linear) rate of $10^{4}$ along the FEAST subspace iterations (while the eigenvalues should converge with a linear rate of $\left.\left(10^{4}\right)^{2}=10^{8}\right)$.

It is important to note that the same convergence rate applies to any arbitrary intervals $[a, b]$ if one chooses $M_{0}$ equal to the eigenvalue count inside a larger interval $[a-\alpha, b+\alpha]$ with $\alpha=(b-a) / 4$. This eigenvalue count within the larger interval can be estimated, in turn, using a reduced number of integration points that can take advantage of iterative solvers with modest residuals (as discussed in Sec. 5.5). The results obtained in Table 2 for the Na5 example, illustrate how an appropriate estimate on a larger interval can be used by FEAST to guarantee a certain degree of convergence rate for the interior eigenpairs. In particular, the results show a linear convergence rate for $n_{c}=8$ in agreement with the expected $4 \times 10^{4}$ that can be directly obtained from a reading of the data in Fig. 2 (at $\lambda= \pm 1.5$ ). Moreover, the value of the rational function using $n_{c}=5$ indicates an expected convergence rate of 


\begin{tabular}{c|cll|cll}
\multicolumn{9}{c}{$n_{c}=8$} & \multicolumn{3}{c}{$n_{c}=5$} \\
\hline \hline Iteration & \# eigenvalue & residual & conv. rate & \# eigenvalue & residual & conv. rate \\
0 & 62 & $1.96 \times 10^{-1}$ & & 65 & $2.06 \times 10^{-1}$ & \\
1 & 59 & $1.58 \times 10^{-7}$ & & 59 & $1.18 \times 10^{-3}$ & \\
2 & 59 & $8.78 \times 10^{-11}$ & $1.8 \times 10^{4}$ & 59 & $3.89 \times 10^{-5}$ & $3.0 \times 10^{1}$ \\
3 & 59 & $1.46 \times 10^{-14}$ & $6.0 \times 10^{4}$ & 59 & $6.48 \times 10^{-8}$ & $6.0 \times 10^{2}$ \\
4 & & & & 59 & $1.30 \times 10^{-11}$ & $5.0 \times 10^{3}$ \\
5 & & & 59 & $5.02 \times 10^{-14}$ & $2.6 \times 10^{2}$ \\
\hline \hline
\end{tabular}

Table 2: Convergence results obtained using FEAST v2.1 for a new interval $[a, b](a=$ 1.38695.. and $b=1.88290 .$.$) containing 59$ eigenvalues and where the subspace size has been estimated at 100 by counting the eigenvalues between $[a-(b-a) / 4, b+(b-a) / 4]$. Two runs using $n_{c}=8$ and $n_{c}=5$ integration points are considered, and the convergence rates are provided from the moment the number of interior eigenvalues stabilizes.

$2 \times 10^{2}$ while the reported rates in Table 2 appears to be in agreement or much better after few iterations.

\subsection{Rational approximation filtering for non-symmetric problems}

Since the eigenvalues of any projector $P$, whether orthogonal or not, are equal to either zero or one, its trace is always equal to the number of its nonzero eigenvalues, which in the case under consideration is the number of eigenvalues located inside the contour integral. The stochastic estimator also works for non-symmetric matrices. Therefore, the whole technique extends to non-Hermitian case without any difference. The contour integration approach for computing the eigenpairs in a given region of the complex plane has also been successfully utilized within the framework of the FEAST solver [15].

Here we present some preliminary results on the applicability of the eigenvalue count estimate (19) for non-symmetric problems. In order to illustrate the accuracy of this approach, we select the complex symmetric matrix 'qc324' available from the Matrix Market 4 which is of size $n=324$. Using a circle centered at $(0,0)$ with radius 0.04 , the exact (complex) eigenvalue count is 37 . In order to estimate the $\operatorname{trace} \operatorname{tr}(P)$ one generates complex random vectors $v_{k}, k=1, . ., n_{v}$ with entries \pm 1 for both the real and imaginary parts. The imaginary part of the trace is expected to be very small, so the estimates are obtained using the real part of the trace in our simulation results. Fig. 8 presents the estimates obtained using two different contour integrations. One notes that $n_{c}=6$ (i.e. Gauss-3 for each half-circle) already provides some reasonable estimates. Further work would be needed to report a detailed study of the accuracy of the approach, but this preliminary result shows promise on the potential extension of the estimated eigenvalue counts to the complex plane.

\footnotetext{
${ }^{4}$ http://math.nist.gov/MatrixMarket/
} 


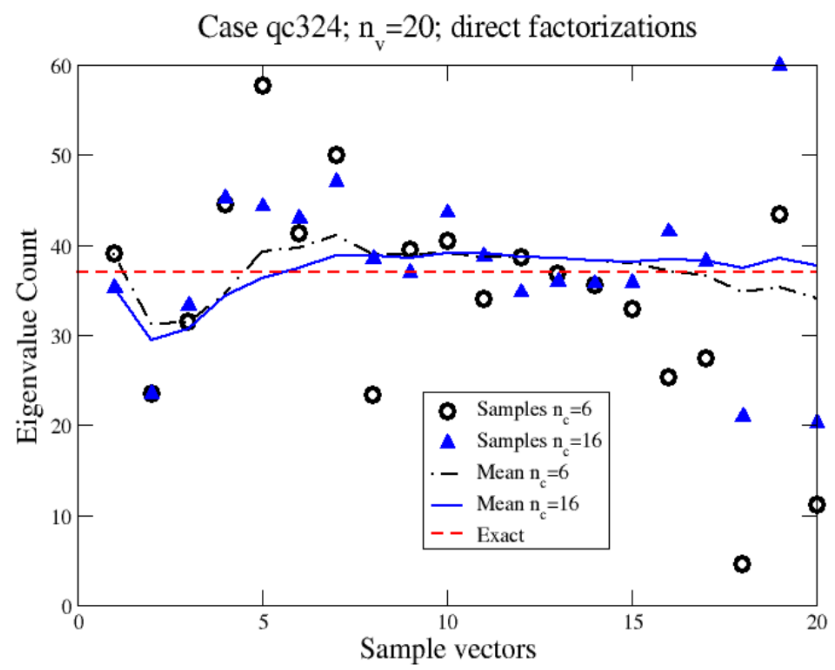

Figure 8: The rational approximation method at work for the qc324 matrix using a direct solver. The plots compare a run using 6 and 16 integration points for the whole contour (obtained by placing respectively 3 and 8 Gauss integration points in the half-circle) and 20 sample vectors.

\section{Conclusion}

The methods presented in this paper rely on a compromise between accuracy and speed. If one is interested in an exact count, then clearly combining the Sylvester inertia theorem with some direct solution method may be the best option, although this may be too costly or even impractical in some situations. Otherwise, a stochastic estimate based on approximating the trace of the eigen-projector by exploiting a polynomial or rational function expansion, will be sufficient. The rational approximation viewpoint may be perfectly suitable within the context of a package like FEAST since (approximate) factorizations will be needed at the outset anyway. The initialization of the package will begin by estimating the eigenvalue count in order to determine the proper subspace size $M_{0}$ to use. The additional cost of this step then remains relatively small and its use may lead to great savings. In other applications the polynomial approximation can give a good estimate at a relatively low cost.

Since the proposed methods are all based on an approximation of the spectral projector, they are subject to a slight bias if this approximation is not accurate enough. This bias may be exacerbated by the presence of clusters near the interval boundaries, since it is generally near these locations that the inaccuracies of the approximate projector are large. Finding reliable methods to reduce this bias remains an open issue that is worth investigating.

\section{References}

[1] A. Angot, Complements de Mathematiques, Editions de la revue d'optique, Paris, France, 1949.

[2] H. Avron And S. Toledo, Randomized algorithms for estimating the trace of an implicit symmetric positive semi-definite matrix, Journal of the ACM, 58 (2011), p. 8. 
[3] C. Bekas, E. Kokiopoulou, And Y. SAAD, An estimator for the diagonal of a matrix, Applied Numerical Mathematics, 57 (2007), pp. 1214 - 1229. Numerical Algorithms, Parallelism and Applications (2).

[4] T. A. DAvis, Direct methods for sparse linear systems, SIAM, Philadelphia, PA, 2006.

[5] FEAST solver, 2009-2013. http://www.feast-solver.org.

[6] Y. Futamura, H. Tadano, and T. Sakurai, Parallel stochastic estimation method of eigenvalue distribution, Japan Society for Industrial and Applied Mathematics (JSIAM) Letters, 2 (2010), p. 127130.

[7] J. A. George And J. W. Liu, Computer Solution of Large Sparse Positive Definite Systems, Prentice-Hall, Englewood Cliffs, NJ, 1981.

[8] G. H. Golub And C. F. V. Loan, Matrix Computations, Johns Hopkins University Press, Baltimore, MD, 3rd ed., 1996.

[9] N. Halko, P. Martinsson, And J. Tropp, Finding structure with randomness: Probabilistic algorithms for constructing approximate matrix decompositions, SIAM Review, 53 (2011), pp. 217-288.

[10] M. F. Hutchinson, A stochastic estimator of the trace of the influence matrix for Laplacian smoothing splines, Commun. Statist. Simula., 19 (1990), pp. 433-450.

[11] T. Iitake And T. EBisuzaki, Random phase vector for calculating the trace of a large matrix, Phys. Rev. E, 69 (2004), p. 057701.

[12] L. O. JAY, H. Kim, Y. SAAD, And J. R. Chelikowsky, Electronic structure calculations using plane wave codes without diagonlization, Comput. Phys. Comm., 118 (1999), pp. 21-30.

[13] L. Krämer, E. D. Napoli, M. Galgon, B. Lang, and P. Bientinesi, Dissecting the FEAST algorithm for generalized eigenproblems, Journal of Computational and Applied Mathematics, 244 (2013), pp. 1 - 9.

[14] C. Lanczos, Applied Analysis, Dover, New York, 1988.

[15] S. LAux, Solving complex band structure problems with the FEAST eigenvalue algorithm, Phys. Rev. B, 86 (2012), p. 075103.

[16] L. Lin, C. YAng, AND Y. SAAD, Approximating spectral densities of large matrices, Tech. Rep. ys-2013-1, Dept. Computer Science and Engineering, University of Minnesota, Minneapolis, MN, 2013. In preparation - to be submitted to SIAM J. Scientific Comput.

[17] L. Lopez And V. Simoncini, Analysis of projection methods for rational function approximation to the matrix exponential, SIAM Journal on Numerical Analysis, 44 (2006), pp. 613-635. 
[18] Y. Maeda, Y. Futamura, And T. SAKurai, Stochastic estimation of eigenvalue density for nonlinear eigenvalue problem on the complex plane, JSIAM Letters, 3 (2011), pp. $61-64$.

[19] M. W. Mahoney, Radomized Algorithms for Matrices and Data, NOW Publishers Foundations and Trends in Machine Learning series, 2011. Also available in arXiv 1104.5557.

[20] E. POLIZZI, A density matrix-based algorithm for solving eigenvalue problems, phys. rev. B, 79 (2009).

[21] E. POLIZZI, A high-performance numerical library for solving eigenvalue problems. arXiv:1203.4031, 2013.

[22] T. J. Rivlin, An Introduction to the Approximation of Functions, Dover, 1969.

[23] H. Roder, R. Silver, D. Drabold, and J. Dong, The kernel polynomial method for non-orthogonal electronic structure calculation of amorphous diamond, Phys. Rev. B, 55 (1997), pp. 15382-15385.

[24] Y. SAAD, Filtered conjugate residual-type algorithms with applications, SIAM Journal on Matrix Analysis and Applications, 28 (2006), pp. 845-870.

[25] — Numerical Methods for Large Eigenvalue Problems- classics edition, SIAM, Philadelpha, PA, 2011.

[26] T. Sakurai And H. Sugiura, A projection method for generalized eigenvalue problems using numerical integration, Journal of Computational and Applied Mathematics, 159 (2003), pp. $119-128$.

[27] G. Schofield, J. R. Chelikowsky, and Y. SaAd, A spectrum slicing method for the kohn-sham problem, Computer Physics Communications, 183 (2012), pp. 497-505.

[28] K. Senzaki, H. Tadano, T. Sakurai, And Z. Bai, A method for profiling the distribution of eigenvalues using the AS method, Taiwanese Journal of Mathematics, 14 (2010), pp. 839-853.

[29] R. Silver, H. Roeder, A. Voter, And J. Kress, Kernel polynomial approximations for densities of states and spectral functions, Journal of Computational Physics, 124 (1996), pp. $115-130$.

[30] R. N. Silver AND H. RÖDER, Densities of states of mega-dimensional Hamiltonian matrices, Int. J. Mod. Phys. C, 5 (1994), pp. 735-753.

[31] J. M. TANG AND Y. SAAD, A probing method for computing the diagonal of a matrix inverse, Numerical Linear Algebra with Applications, 19 (2011), pp. 485-501.

[32] P. T. P. TANG AND E. Polizzi, FEAST as a subspace iteration eigensolver accelerated by approximate spectral projection, SIAM Journal on Matrix Analysis and Applications, 35 (2014), pp. 354-390. 
[33] L. W. WANG, Calculating the density of sates and optical-absorption spectra of large quantum systems by the plane-wave moments method, Phys. Rev. B, 49 (1994), pp. 10154-10158.

[34] M. N. Wong, F. Hickernell, And K. Liu, Computing the trace of a function of a sparse matrix via hadamard-like sampling, Tech rep. 377(7/04), Hong Kong Baptist University., (2004). 